\title{
DETC2017-68306
}

\section{A SMART DEVICE TO SUBSTITUTE THE NEUROTHESIOMETER}

\author{
Neil Vaughan, Venketesh N. Dubey \\ Bournemouth University \\ Faculty of Science and Technology \\ Fern Barrow, Poole, BH12 5BB, United Kingdom
}

\author{
Tamas Hickish, Jonathan Cole \\ Poole Hospital NHS Foundation Trust, \\ Longfleet Rd, Poole, Dorset, \\ BH15 2JB, United Kingdom
}

\begin{abstract}
This paper presents a patented smart point-of-care testing (POCT) system for the diagnosis and grading of peripheral neuropathy at the patient's home or care center. The device aims to detect changes or worsening of a patient's neuropathy.

Our system utilizes the vibration motor within a smartphone, applied through a 3D printed probe attachment to detect sensation loss in vibration sensitivity threshold (VST). A smartphone app displays several neuropathy questionnaires to the user to identify and monitor changes in their condition.

This paper presents results from comparison between the new smart device and the gold standard Neurothesiometer. Results suggest that the new device performs closely to the gold standard in terms of the frequency and amplitude of vibration.
\end{abstract}

\section{Keywords: Neuropathy, Diagnosis, Smartphone App.}

\section{INTRODUCTION}

Peripheral neuropathy is a nerve damage condition. The number of patients suffering is large including $10 \%$ of over $55 \mathrm{~s}$. This commonly occurs in patients suffering from cancer or diabetes, two of the most common diseases worldwide.

Diabetes affects 3.8 million people in the UK $(7.4 \%$ of the population). Of all diabetes patients, $60 \%$ suffer peripheral neuropathy, causing loss of sensation in fingers and toes.

This POCT neuropathy diagnosis system avoids the requirement for patients to travel to a hospital, freeing consultation time for clinicians, using wireless data to provide a comprehensive objective assessment and detect changes in the patient's condition. Resulting data is transferred to clinicians through an online patient portal database which tracks the history of each patient's condition over time.

Our smart device aims to identify warning signs of neuropathy to provide indication of a patient beginning to suffer from diabetic limb loss. Neurosthesiometers and other Neurologic screening tests such as $10 \mathrm{~g}$ monofilament and biothesiometers have previously been used as the gold standard to identify and monitor the progression of neuropathy, and to identify the dosage of preventive strategies. The current gold standard Neurothesiometer produces a vibration with frequency fixed at $110 \mathrm{~Hz}$ and the amplitude is adjustable by the user controls.

POCT has been used for other conditions such as haemostatic dysfunction, gonorrhea or HIV [1]. Previous POCT neuropathy devices have been simple, not incorporating any questionnaires or online data collection, limiting their use. The VibraTip (McCallan Medical) is a handheld key-fob device to test vibration sensitivity. This was found to be comparable to the neurothesiometer, and superior to the tuning fork [2].

Alternative devices include the $128 \mathrm{~Hz}$ electronic tuning fork (ETF) [3], thermal stimulus [4] or ultrasound scanning of fibers. A patent was filed for a vibration pen pocket device utilized for detecting severe neuropathy [5]. The device is shaped like a pen including a stimulus probe mounted at the tip. The probe vibrates and is applied to a patient's extremity in order to determine if the patient can feel the vibration.

The clinical requirement for a neuropathy device is to test a wide range of sensation on the patient's skin, so that very low vibration should be possible, for patients who are only beginning to suffer sensation loss. This requires the device to have full control of the vibration strength onto the skin surface.

Human sensation of vibration is operated through small fibers of sensation. These small fibers should be activated at the frequency of device vibration. The frequency of fiber activation is known through previous literature [6].

\section{DESIGN AND DEVELOPMENT}

In response to the lack of suitable point-of-care solutions for the testing of peripheral neuropathy, we have designed the "NEuRopathy evaluation using the Vibration function of a mobile phonE" (NERVE) device. Two patents have been filed for the NERVE device in UK and Internationally (see acknowledgements). The design consists of a smartphone app and also a 3D printed attachment. The design steps are shown in the following sections.

Our NERVE device uses a smartphone vibration motor. Mobile devices commonly vibrate at $130 \mathrm{~Hz}-180 \mathrm{~Hz}$, an average $160 \mathrm{~Hz}(9600 \mathrm{rpm})$ based on analysis of the motor manufacturer specifications [7]. The optimum frequency for the best vibrotactile perception for static state is $151 \mathrm{~Hz}$ [7]. 
An app was produced which is compatible with many popular mobile phones, tablets and devices. This enables any patient with a mobile device to use the developed app at their home unaided. For our initial proof of concept system, the Android mobile operating system was used with a Samsung Galaxy Fame smartphone.

\section{GEOMETRIC 3D SCANNING THE MOBILE DEVICE}

Initially the 3D geometry of the mobile device needed to be measured, so that we could design an attachment to exactly match the shape of the mobile phone. Laser scanning of the 3D volume of the phone was completed using an optical scanner which captured the precise size and shape of the mobile phone contours on both sides and the top. Laser scanning ensures that the attachments fit perfectly onto the phone. Fig. 1. Shows the raw volume data obtained from the 3D laser scan. There are several imperfections in the data, due to the reflective areas of the mobile device which relected the laser beams. Fine chalk dust was aplied to the surface to improve the laser detection on reflective areas.

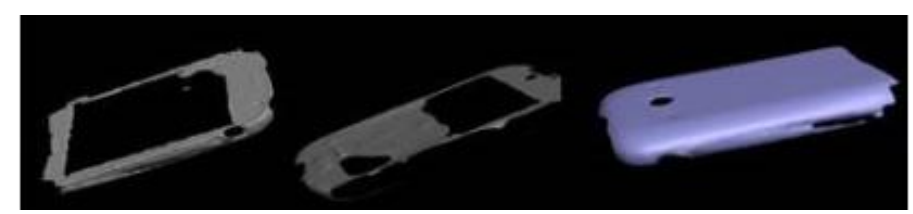

Fig. 1. Raw data from 3D Laser scan.

\section{MODELLING THE CAD ATTACHMENT}

Computer aided design (CAD) models were created matching the external geometrical shape of the phone. A uniform $3 \mathrm{~mm}$ wall thickness was used to ensure consistency. The volume of the mobile phone was subtracted from a block so that the phone fits perfectly into the resulting cavity. The attachments were designed to connect onto the top or side of the phone. Three main designs were printed (Fig. 2.): (i) A $36 \mathrm{~mm}$ pointed spike. (ii) A rod with a rounded end matching diameter of the neurosethisomer. (iii) A helix having some spring properties for enabling resonance and damping. Once the CAD models were completed they were converted into STL format for 3D printing.

The attachments aim to apply the vibration in a controlled way through a tip which is shaped in the optimal way to transfer the vibration according to predefined mechanical vibration characteristics.

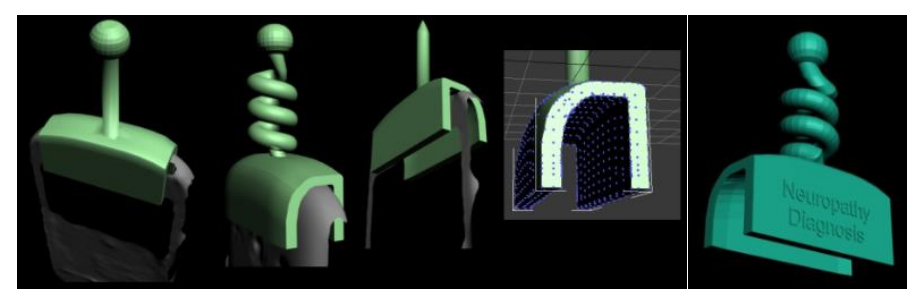

Fig. 2. Three CAD vibration attachments for 3D printing.

\section{THE 3D PRINTING OF THE PROBE}

The parts were manufactured on two different 3D printing machines for comparison: Stratasys and Makerbot (Fig. 3). The CAD models had been designed using a true 1:1 scale in the software. Precision caliper measurements were taken from the mobile device and compared to measurements from 3D scanning to ensure correct thickness measurements. Due to this, the parts were printed using the size scales defined within the CAD software, so that they fitted perfectly onto the mobile device. Using this method the $3 \mathrm{D}$ printed probe fits tightly onto the top of the phone, holding on by its own friction grip.

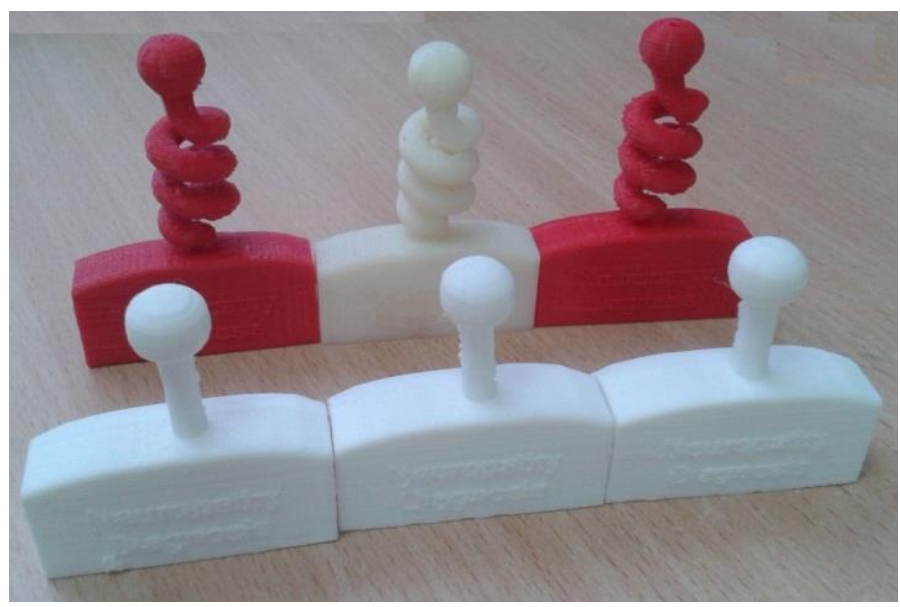

Fig. 3. The range of 3D printed phone attachments.

A close contact between the device and attachment is vital. It is important that the metallic rim of the mobile phone device comes into direct and full contact with the solid part of the attachment, to ensure that the full vibration is transferred from the phone into that attachment. The metal rim of the mobile device transfers the vibration, as the plastic phone cover does not fully vibrate with the phone vibration.

The current 3D printed devices grip onto the mobile device using friction from their precise thickness. However we have also designed a one-fits-all attachment for patients owning several mobile devices of varying thickness. Two one-fits-all attachments were designed which enable the attachment to fit various device thicknesses, using a screw to tighten the attachment onto the device or a bulldog clip design.

A benefit of using plastic ABS material for the attachments is that they are cheap and disposable, so they could be given to the patients, or sold with little cost. Plastics have aesthetic temperature qualities as opposed to metal which is cold against the skin, which may cause patient discomfort. The initial parts were 3D printed for rapid manufacturing, however if mass produced, a range of alternative production approaches could ensure cheaper mass production. For mass production these initial 3D printed prototypes would serve as a mold for larger scale milling or injection molding to yield cheaper unit pricing. 


\section{APP SOFTWARE USER INTERFACE DESIGN}

The software system uses a smart-phone app to assess the patient's neuropathy condition. The app could be used with all popular models of mobile phones, tablets and devices. This could enable any patient with a mobile device to use the developed app at their home unaided. For our initial proof of concept system, the Android mobile operating system was used since it has the largest installed base of any OS, with over 1 billion active monthly users, and is selling more than Windows, iOS and Mac OS combined. The smartphone used is a Samsung Galaxy Fame, which is commonly available since Samsung currently sell more smart-phones than all other manufacturers combined [8].

Existing neuropathy questionnaires from clinical literature were programmed into a series of simplified data screen in the app. The user is asked whether they feel worsening sensations in their hands, feet, toes, such as tingling, numbness, and burning. This could include temperature change, burning, stabbing and cramping in arms, hands. A screen requests the user to indicate which common activities they are experiencing increased trouble with achieving unaided, such as doing up buttons, tying shoelaces, cooking, using cutlery and others. An algorithm was devised which takes into account the patient's answers to these questions to produce a final score and grading of neuropathy. This algorithm may help to grade neuropathy, or could refer patient responses back to the clinician to produce a grading.

A series of screens were devised, aiming to minimize complexity taking into account elderly patients may be unfamiliar with use of apps (Fig. 4). The screens are (i) front page, (ii) screen for entering name and age. (iii) a questionnaire regarding sensation changes, (iv) questions regarding difficulties experienced during common everyday activities, (v) select vibration for hands, feet of knees, (vi) a diagram with video showing instructions for attaching the probe and holding the device (vii) the screen during which vibration occurs and the user can select whether they feel a sensation (viii) A results screen displaying the patient name, age, VST, results, GPS location.

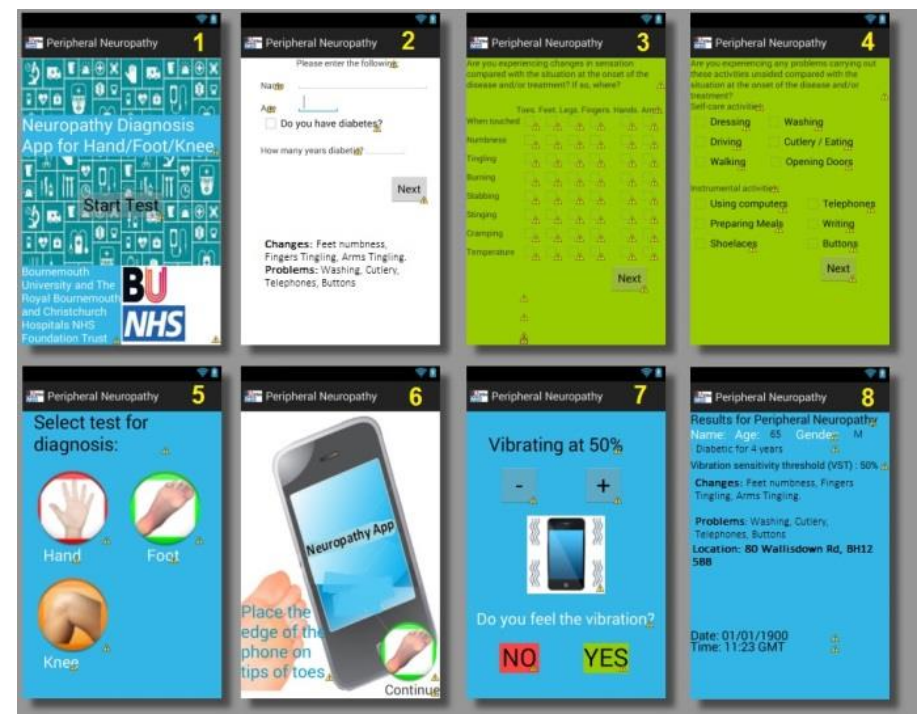

Fig. 4. Screens designed for user interface.
Data from geostationary positioning satellite (GPS) and wireless network data is used to identify a street address of the patient's current location. This is sent back to the clinicians, who are keen to use GPS locations to analyse links between geographical location and neuropathy, especially if the app is used over a wide geographical population.

The app asks the user for their age and how many years they have had diabetes (if applicable). This data can be used to detect any link between age and neuropathy.

When the vibration motor is started on screen 7 (Fig. 4), the user is asked whether they feel the vibration and can answer yes or no. If no is selected, the vibration strength increases. When the user subsequently answers yes, they feel a vibration, that current level of vibration is stored as the patient's current vibration sensitivity threshold (VST).

The NERVE device with the app running on the mobile phone and attachment is shown in Fig. 5.

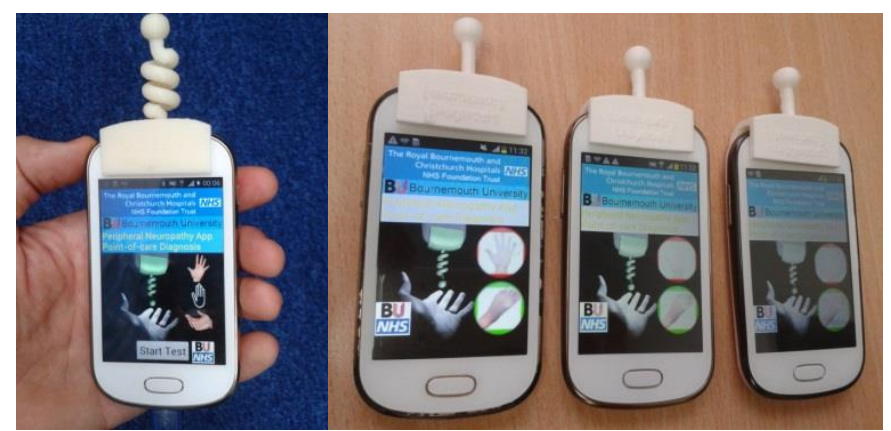

Fig. 5. Complete POCT neuropathy diagnosis system.

\section{DYNAMIC VIBRATION MODULATION}

An important aspect of testing neuropathy is to adjust the vibration strength, so that individuals of a wide range of sensitivities can be tested. This is achieved in the NERVE device by adjusting vibration strength in the App and by the design of the probe to transmit to vibration onto the patient's skin. The modulation steps and vibration testing steps are described in this section.

\section{BACKGROUND OF MOBILE VIBRATION MOTORS}

Vibration motors inside mobile phones are used as a haptic method to alert the user without using any audio output. Since the aim of mobile vibration function is usually to simply produce any noticeable sensation to alert the user, a full characterization of the vibrations emitted from mobile devices in terms of amplitude and frequency may not previously have been necessarily completed during manufacture.

Vibration motors come in various designs depending on manufacturer of the mobile device. The method of vibration used within vibration motors is an offset mass mounted onto the motor axle. Vibration motors have several variables: (i) axle length (ii) mass of the offset (iii) offset length (iv) supply voltage. The output vibration can be characterized in terms of (i) displacement or amplitude, (ii) force amplitude, (iii) frequency and (iv) electrical parameters. Previous research found that when 
approaching resonance, power increases drastically while resistance drops.

Various vibration motor designs are in use in mobile devices. Samsung Galaxy S3 and Sony Xperia use flat coin vibration motors with net weight of $6 \mathrm{~g}$. Alternatively iPhone uses a cylinder motor design. The offset mass can be either circular or semi-circular. The axle diameter can range from $1 \mathrm{~mm}$ to $4 \mathrm{~mm}$, external diameter from $3 \mathrm{~mm}$ to $5 \mathrm{~mm}$. Operating voltage varies on a range of 2.5-3.8V for vibration motors. The required current for the coin motor is roughly 0.033 amps per volt supplied, which equates to around $0.1 \mathrm{~A}$ for a $3 \mathrm{~V}$ motor.

When the vibration motor spins it exerts the vibrational force mainly on two axes which are the axes directions towards which the eccentric mass move. The attachment should be mounted on one of these two axes. Over time if the voltage and amplitude supplied to the motor varies, both the frequency and amplitude change. At high motor speeds, other aspects such as the flexion in the motor shaft will also affect the vibration.

\section{VIBRATION MODULATION IN THE NERVE APP}

Mobile phones do not natively provide any hardware or software methods to control the strength of motor vibration, only on and off controls are provided. There are recently some haptic effect apps designed for smartphones which can produce various haptic effects, primarily used in games, for example shooting or driving. Those haptic effects were not suitable for our device, which requires fine-tuned control.

In order to modulate the phone's natural vibration frequency, a software app was programmed to control the motor speed. Within our developed app, a software method was developed to control the motor power. Controlling the vibrator power through software caused simultaneous adjustment of both frequency and amplitude of vibration, although one cannot be controlled independently of the other, since this is inherent to the device. This is similar to harmonic force vibration [9].

The lowest possible programmable vibration level of the phone is described as $20 \%$. If any less than $20 \%$ power is supplied, then the motor physically stops turning.

\section{MEASUREMENT OF THE NERVE DEVICE VIBRATION}

The aim of the vibration measurement test was to compare the NERVE device to the current gold standard Neurothesiometer device. The experiments were done by comparing amplitude and frequency of the two devices. Frequency $(\mathrm{Hz})$ was measured by audio analysis, because the audio frequency matches mechanical frequency, a principle used by the tuning fork. Amplitude $(\mu \mathrm{m})$ was calculated indirectly by measuring the acceleration $\left(\mathrm{m} / \mathrm{s}^{2}\right)$ using accelerometers and then using an equation to convert frequency and acceleration into displacement $(\mu \mathrm{m})$.

The results show that the NERVE device is equivalent to the Neurothesiometer. The NERVE device should be used on settings $22 \%-26 \%$, which produce frequency $100-135 \mathrm{~Hz}$ and amplitude $1.3 \mu \mathrm{m}$. This is equivalent to the neurothesiometer setting $4(28 \mathrm{~V})$, which produces frequency $110 \mathrm{~Hz}$ and amplitude
$1.3 \mu \mathrm{m}$. This ensures the frequency stays within the ideal range for human perception.

\section{ACCELEROMETER MEASUREMENTS}

Accelerometers were used to record the motion of the NERVE device (Fig. 6) and neurothesiometer (Fig. 7) when vibrating at each setting. Using this recorded accelerometer data, the maximum accelerations were measured for each NERVE setting in Table 1 and for Neurothesiometer in Table 2.

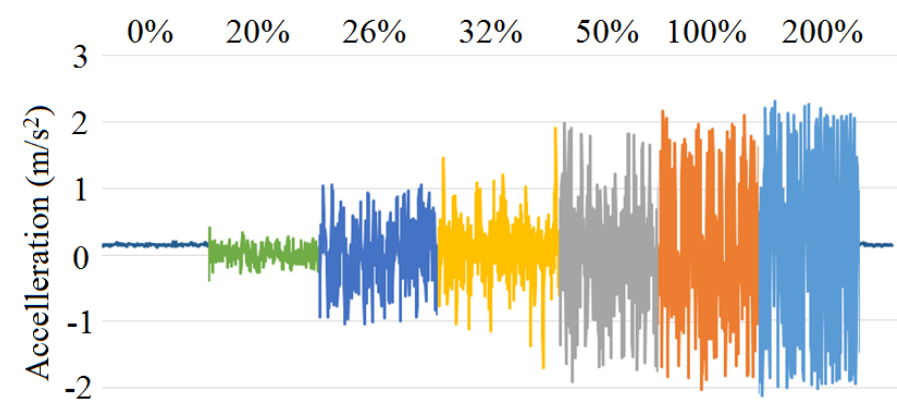

0123456789101112131415161718192021

Time (seconds)

Fig. 6. NERVE device Accelerometer data for each setting.

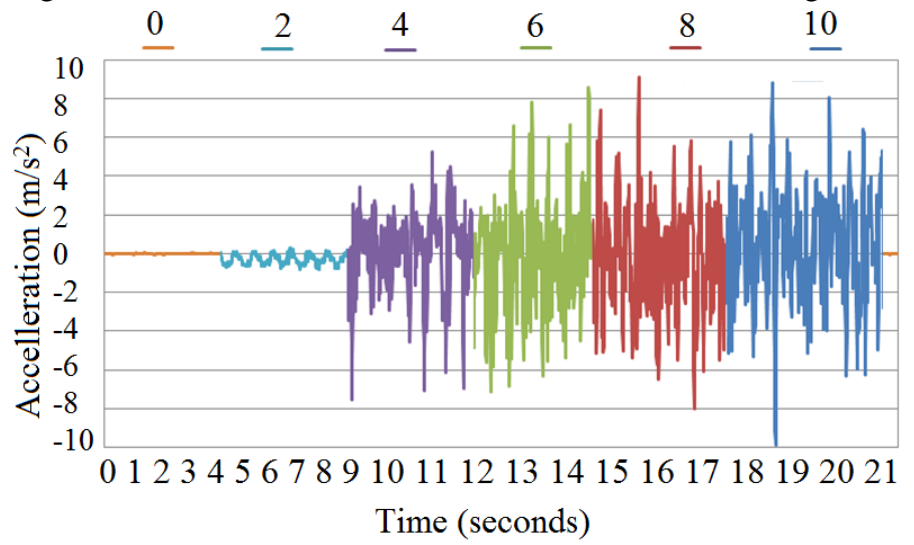

Fig. 7. Neurothesiometer Accelerometer data for each setting.

\begin{tabular}{|l|l|r|r|r|r|r|r|}
\hline & $0 \%$ & $20 \%$ & $26 \%$ & $32 \%$ & $50 \%$ & $100 \%$ & $200 \%$ \\
\hline $\max$ & 0.20 & 0.43 & 1.19 & 1.91 & 2.10 & 2.29 & 2.48 \\
\hline & & - & - & - & - & & \\
min & 0.09 & 0.42 & 1.05 & 1.76 & 1.97 & -2.23 & -2.25 \\
\hline range & 0.10 & 0.85 & 2.25 & 3.68 & 4.07 & 4.53 & 4.73 \\
\hline
\end{tabular}

Table 1. NERVE device Acceleration $\left(\mathrm{m} / \mathrm{s}^{2}\right)$ - Min and Max over 20 seconds.

\begin{tabular}{|l|l|l|l|l|l|l|}
\hline & $\begin{array}{l}0 \\
(0 \mathrm{~V})\end{array}$ & $\begin{array}{l}2 \\
(20 \mathrm{~V})\end{array}$ & $\begin{array}{l}4 \\
(28 \mathrm{~V})\end{array}$ & $\begin{array}{l}6 \\
(36 \mathrm{~V})\end{array}$ & $\begin{array}{l}8 \\
(44 \mathrm{~V})\end{array}$ & $\begin{array}{l}10 \\
(49 \mathrm{~V})\end{array}$ \\
\hline $\max$ & 0.045 & 0.316 & 5.245 & 8.559 & 9.112 & 8.789 \\
\hline & - & - & & & & \\
$\min$ & 0.062 & 0.924 & -7.544 & -8.034 & -8.439 & -9.892 \\
\hline range & 0.107 & 1.240 & 12.789 & 16.594 & 17.551 & 18.681 \\
\hline
\end{tabular}

Table 2. Neurothesiometer Acceleration $\left(\mathrm{m} / \mathrm{s}^{2}\right)$ - Min and Max over 20 seconds. 


\section{FREQUENCY MEASUREMENT BY AUDIO ANALYSIS}

Unfortunately the accelerometer recordings could not identify the frequency $(\mathrm{Hz})$, because the fastest possible rate the accelerometers could be recorded was a sampling rate of $100 \mathrm{~Hz}$. This is not fast enough to cover the expected $160 \mathrm{~Hz}$ range of mobile phone vibration motors.

To solve this problem, audio analysis was used to measure frequency. The frequency of mechanical vibration is equal to the frequency of audio that it generates, which is the principle used in a tuning fork. Audio recordings were captured using a microphone. Audio was recorded for all vibration strengths. The audio was recorded at a sample rate of $44.1 \mathrm{KHz}$, which is fast enough to capture vibrations in the range of $160 \mathrm{~Hz}(0.16 \mathrm{KHz})$. For each vibration strength, exactly $100 \mathrm{~ms}$ audio sample was analysed, to count the number of waves which occurred. Audio was recorded separately for each neurothesiometer strength setting: $2,4,6,8,10$, which equate to voltages of $20 \mathrm{~V}, 30 \mathrm{~V}, 36 \mathrm{~V}$, $45 \mathrm{~V}, 49 \mathrm{~V}$. Audio recordings are visualised as a waveform for NERVE device in Fig. 8 and Neurothesiometer in Fig. 9.

NERVE device frequency measurement results (Table 3), show that frequency increased as vibration strength increased, from $90 \mathrm{~Hz}(20 \%)$ to $215 \mathrm{~Hz}(200 \%)$.

Neurothesiometer frequency measurement results (Fig. 9) show that as vibration strength setting was increased from 2, 4, 6,8 and 10, the frequency remained constant at $110 \mathrm{~Hz}$.

For both devices, the amplitude increased as the vibration strength increased, but in the Fig. 8 and Fig. 9, the amplitudes have been normalised to the same vertical scale, to make it easier to visually locate the wave peaks. The original time scale is preserved on $\mathrm{x}$ axis. Increased audio amplitude does not imply increased mechanical vibration amplitude, which is why this audio data was used to measure frequency, not amplitude.

\begin{tabular}{|l|l|l|l|}
\hline $\begin{array}{l}\text { Speed } \\
(\%)\end{array}$ & $\begin{array}{l}\text { Peaks in 0.1 } \\
\text { seconds }\end{array}$ & $\begin{array}{l}\text { Time period } \\
(\mathrm{ms})\end{array}$ & $\begin{array}{l}\text { Frequency } \\
(\mathrm{Hz})\end{array}$ \\
\hline $12 \%$ & Unmeasurable & Unmeasurable & Unmeasurable \\
\hline $16 \%$ & Unmeasurable & Unmeasurable & Unmeasurable \\
\hline $20 \%$ & 9 & 11.4 & 90 \\
\hline $22 \%$ & 10 & 10 & 100 \\
\hline $23 \%$ & 11 & 9.1 & 110 \\
\hline $24 \%$ & 12 & 8.3 & 120 \\
\hline $25 \%$ & 13 & 7.7 & 130 \\
\hline $26 \%$ & 13.5 & 7.4 & 135 \\
\hline $28 \%$ & 15 & 6.6 & 150 \\
\hline $29 \%$ & 16 & 6.2 & 160 \\
\hline $30 \%$ & 17 & 5.8 & 170 \\
\hline $32 \%$ & 17.5 & 5.7 & 175 \\
\hline $34 \%$ & 18 & 5.5 & 180 \\
\hline $36 \%$ & 19 & 5.2 & 190 \\
\hline $42 \%$ & 19.5 & 5.1 & 195 \\
\hline $50 \%$ & 20.5 & 4.9 & 205 \\
\hline $100 \%$ & 21 & 4.7 & 210 \\
\hline $200 \%$ & 21.5 & 4.6 & 215 \\
\hline
\end{tabular}

Table 3. NERVE device - Frequency of each vibration strength determined by audio analysis.

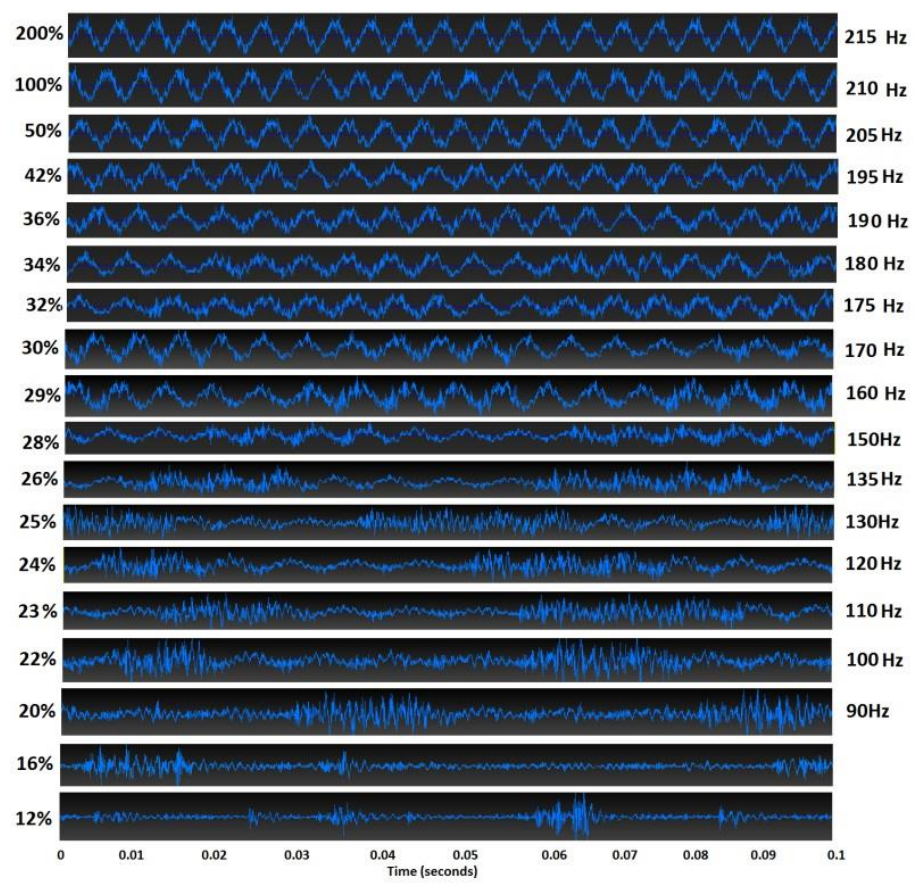

Fig. 8. NERVE device audio recordings showing vibration frequency changes on all strength settings.

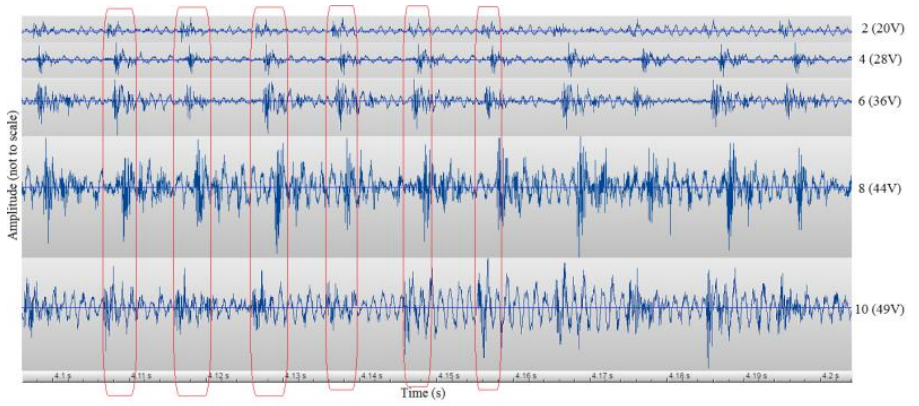

Fig. 9. Neurothesiometer Audio recordings showing vibration frequency at all settings. The red ovals show frequency peaks constant at $110 \mathrm{~Hz}$ on all settings.

\section{AMPLITUDE CALCULATIONS USING EQUATION}

The amplitude of vibration was calculated using Equation 1, as the displacement $x$ based on the known acceleration $a$ and frequency $f$ :

$x=\frac{a}{-(2 \pi f)^{2}}$

where $a=$ acceleration in $\mathrm{m} / \mathrm{s} 2, f=$ frequency in $\mathrm{Hz}, x=$ displacement from the central position in $\mathrm{m}$.

The amplitude $(\mathrm{mm})$ is the largest displacement $(\mathrm{mm})$ that occurred during the vibration.

Theoretically the fastest acceleration occurs at the top and bottom of each vibration wave. Therefore when acceleration is at the maximum, the displacement is also at the maximum. In both devices, for each strength setting, the maximum acceleration (Table 1 and 2) and frequency (Table 3) has been 
measured. Eq. 1 was used to calculate the maximum displacement which occurred during the oscillations, which is equal to the vibration amplitude. Calculations of amplitude for NERVE device are in Table 4 and Neurothesiometer in Table 5.

For the Neurothesiometer, because frequency is fixed, the amplitude is linearly related to the acceleration.

As the NERVE device uses an offset mass, the amplitude is mostly constant irrespective of the speed or frequency, because the amplitude directly depends on the length of the offset shaft and the relation between offset mass, and mass of mobile phone. As frequency increases, more centrifugal force is generated but it is changing direction more quickly giving less time to accelerate the mobile phone.

\begin{tabular}{|l|r|r|r|r|r|r|}
\hline & $20 \%$ & $26 \%$ & $32 \%$ & $50 \%$ & $100 \%$ & $200 \%$ \\
\hline $\begin{array}{l}\text { Maximum } \\
\text { Accelerometer } \\
\text { measurement } \\
(a)\left(\mathrm{m} / \mathrm{s}^{2}\right)\end{array}$ & 0.42 & 1.12 & 1.84 & 2.03 & 2.26 & 2.36 \\
\hline $\begin{array}{l}\text { Audio } \\
\begin{array}{l}\text { Frequency } \\
\text { Measurements } \\
(f)(\mathrm{Hz})\end{array}\end{array}$ & 90 & 140 & 180 & 200 & 210 & 210 \\
\hline $\begin{array}{l}\text { Displacement } \\
=\text { Amplitude } \\
\text { in } \\
\text { micrometers } \\
(x)(\mu \mathrm{m})\end{array}$ & 1.34 & 1.45 & 1.44 & 1.29 & 1.30 & 1.36 \\
\hline
\end{tabular}

Table 4. NERVE device - Calculated amplitude of vibration at each setting.

\begin{tabular}{|l|l|l|l|l|l|}
\hline & $\begin{array}{l}2 \\
(20 \mathrm{~V})\end{array}$ & $\begin{array}{l}4 \\
(28 \mathrm{~V})\end{array}$ & $\begin{array}{l}6 \\
(36 \mathrm{~V})\end{array}$ & $\begin{array}{l}8 \\
(44 \mathrm{~V})\end{array}$ & $\begin{array}{l}10 \\
(49 \mathrm{~V})\end{array}$ \\
\hline $\begin{array}{l}\text { Maximum } \\
\text { Accelerometer } \\
\text { measurement } \\
(a)\left(\mathrm{m} / \mathrm{s}^{2}\right)\end{array}$ & 0.053 & 0.620 & 6.394 & 8.297 & 8.775 \\
\hline $\begin{array}{l}\text { Audio } \\
\text { Frequency } \\
\text { Measurements } \\
(f)(\mathrm{Hz})\end{array}$ & 110 & 110 & 110 & 110 & 110 \\
\hline $\begin{array}{l}\text { Displacement } \\
=\text { Amplitude } \\
\text { in } \\
\text { micrometers } \\
(x)(\mu \mathrm{m})\end{array}$ & 0.112 & 1.298 & 13.387 & 17.369 & 18.371 \\
\hline
\end{tabular}

Table 5. Neurothesiometer-Calculated amplitude of vibration at each setting.

\section{SUMMARY OF VIBRATION COMPARISON}

The neurothesiometer has fixed frequency $(110 \mathrm{~Hz})$, whereas the NERVE device has variable frequency $(90 \mathrm{~Hz}-215 \mathrm{~Hz})$.

The NERVE device has fixed amplitude $(\sim 1.3 \mu \mathrm{m})$ whereas the Neurothesiometer has variable amplitude $(0.1 \mu \mathrm{m}-18.3 \mu \mathrm{m})$.

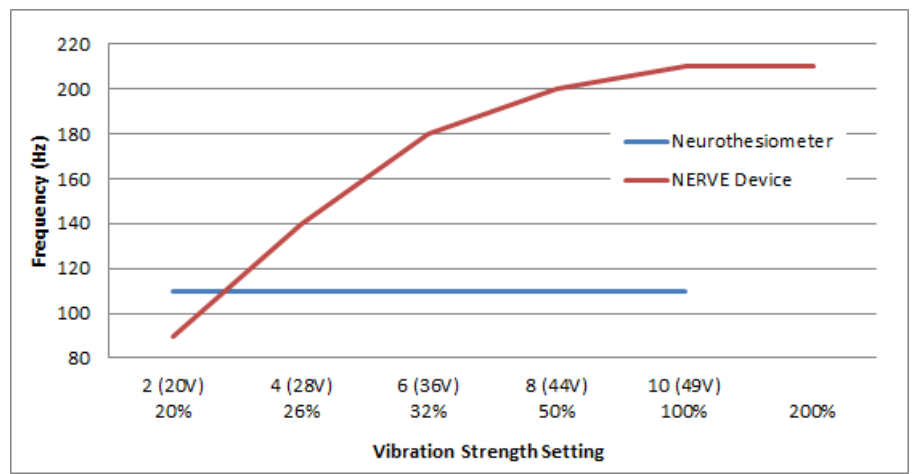

Fig. 10 Frequency $(\mathrm{Hz})$ comparison for NERVE device and Neurothesiometer.

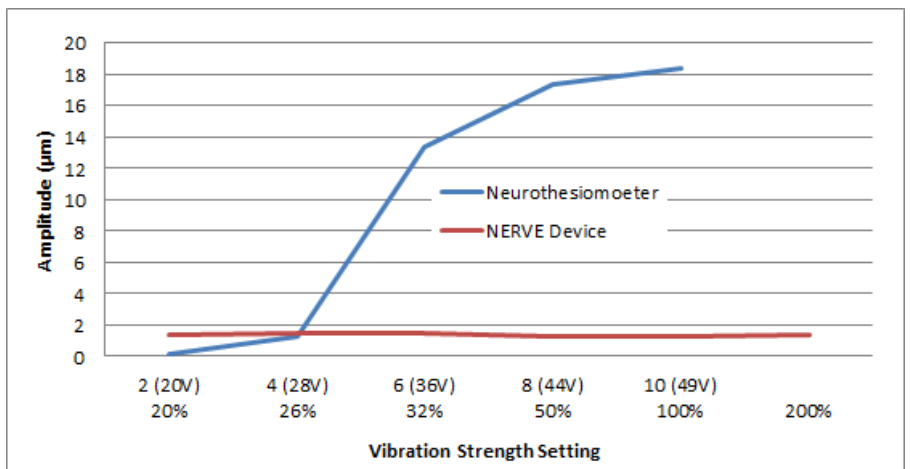

Fig. 11 Amplitude $(\mu \mathrm{m})$ comparison for NERVE device and Neurothesiometer.

The NERVE setting $26 \%$ is closely equivalent to Neurothesiometer setting $4(28 \mathrm{~V})$. There is a crossover point where the NERVE device and neurothesiometer are equivalent in both frequency (Fig. 10) and amplitude (Fig. 11).

The NERVE device setting $26 \%$ has frequency of $140 \mathrm{~Hz}$ which is close to the $110 \mathrm{~Hz}$ Neurothesiometer, close to the Tuning fork frequency of $128 \mathrm{~Hz}$ and within the ideal range for human perception $(100-150 \mathrm{~Hz})$. The NERVE device setting $26 \%$ has Amplitude is $1.3 \mu \mathrm{m}$ which is the same amplitude as the Neurothesimeter at setting $4(28 \mathrm{~V})$.

The point at which the two devices are equivalent is $28 \mathrm{~V}$, is very close to the normal clinical perception test. Clinicians normally consider that patients who cannot sense $25 \mathrm{~V}$ are considered to have neuropathy.

\section{CLINICAL TRIAL TESTING WITH PATIENTS}

The device has been tested on a small number of patients within Royal Bournemouth Hospital NHS Foundation Trust. The data was able to ascertain which levels of vibration various patients can feel. Both the patients and clinicians provided positive feedback. A further clinical trial is planned in the near future to test the phone device on neuropathy patients. The trial will assess whether the algorithm can directly grade neuropathy from the vibration sensitivity, and to test the automatic alerting and referral to a clinician if a patient's performance decreases. 
Initially the gold standard (Neurothesiometer) will be used to evaluate peripheral sensitivity. The protocol for testing with the neurothesiometer will follow the methods suggested by Duke et al (2007). The sensation will be demonstrated first by placing the probe against the shoulder. Participants will be asked to lie supine on bench with bent legs so that feet (socks and shoes are removed) are flat on the bench. The probe will be held at 90 degrees to the skin with constant pressure. Three sites (big toe, ankle and knee) will be used and only the right lower limb will be tested (as recommended by Duke et al, 2007). Testing will begin with the most extreme point (big toe) and voltage will slowly increase from $0 \mathrm{~V}$ at a rate of $1 \mathrm{~V} / \mathrm{s}$ until the participant indicates that they can feel the vibration. This is then repeated after 1 minute break. If the mean detection limit in the toe is less than $50 \mathrm{~V}$, no other sites are tested. For limits greater than $50 \mathrm{~V}$ the process is repeated first at the ankle and then the knee.

This is followed by Autonomic Function Testing (AFT) to separate sensory tests. Following the AFT, the participant's peripheral sensitivity will be evaluated using the NERVE device. First, a demonstration of the vibration will be produced on the participant's shoulder. Following this, the participant will be asked to lie supine on the bench with their feet outstretched. The vibration probe attached to the phone will be held against the fleshy part of the big toe with a constant pressure. The phone's vibration will be increased from $0 \%$ until the participant indicates they have felt the sensation. This level will be recorded by the app. After 1 minute, this will be repeated and the mean $\%$ used as the outcome.

\section{INTERPRETATION}

The aim of this research was to build a point-of-care diagnosis system for peripheral neuropathy. Currently the patient is required to attend the hospital several times to use a neurothesiometer. This system uses an app and vibration motor of the smart phone to offer this functionality in the home setting, saving cost and time. Measurements can be taken more consistently and patient data is accurately recorded.

The 3D printed probe fits tightly onto the top of the phone, which ensures all of the vibration is transferred. The smart-phone attachment fits the phone based on 3D scan data.

The app was used on a small number of patients within Royal Bournemouth Hospital. Initial results were promising. A further structured clinical trial is now being planned. The clinical trial will follow well-define statistical approaches to judge relevance and success of the device.

This is also useful for cancer patients with sensation loss caused by chemotherapy. The device may be useful in third world countries where mobile devices are common and patients are experiencing neuropathy caused by drugs taken for leprosy, AIDS and tuberculosis.

Patients who do not have neuropathy or are at a very early stage but who wish to obtain confirmation of this can also use the app, and only when they score a level below a certain threshold will they then be advised by the app to consult their neuro-physician.
Lower VST and sensation loss may also be caused by prescribed drugs, rather than peripheral neuropathy, such as chemotherapy induced neuropathy or with patients from thirdworld countries taking drugs for tuberculosis.

We aim to continue further evaluation of the device using extracted (physiologically relevant) features from both devices.

\section{ACKNOWLEDGEMENTS}

The authors are grateful to the NHS Acorn Challenge Prize, for funding the clinical trial and 3D printing development. The authors also show their gratitude to the Institution of Engineering \& Technology (IET) for nominating the NERVE device for Innovation Awards. The authors acknowledge NHS Innovations Ltd for supporting the commercialisation of this device. The authors acknowledge Poole Hospital and Royal Bournemouth NHS Foundation Trusts for endorsing and sponsoring this innovation and also organising the proof-of-principle trial, and contributing funds for the patent process. We acknowledge the anonymous reviewers for their constructive feedback.

\section{REFERENCES}

[1] Pearse, B., et al. (2014) Implementing a point of care testing service improves management of haemostatic dysfunction in cardiac surgery patients. Australian Critical Care 27.1: 49.

[2] Nizar, H., Munro, N., Nightingale, P., \& Fehrer, M. D. (2014). Diagnostic accuracy of the VibraTip in detection of diabetic peripheral neuropathy. British Journal of Diabetes \& Vascular Disease, 14(1), 26-29.

[3] O'Brien, T., \& Karem, J. (2014). An Initial Evaluation of a Proofof-Concept 128-Hz Electronic Tuning Fork in the Detection of Peripheral Neuropathy. Journal of the American Podiatric Medical Association, 104(2), 134.

[4] Zatz, Timothy, (2014) 'Neuropathy Test Device', US Patent, US2014163394.

[5] Laudadio, Charles, (1996) 'Method of detecting neuropathy by vibration', US Patent, US5931793.

[6] Lauria, Giuseppe, Ingemar SJ Merkies, and Catharina G. Faber. "Small fibre neuropathy." Current opinion in neurology 25.5 (2012): 542-549.

[7] Yim, J., Myung, R., \& Lee, B. (2007). The mobile phone's optimal vibration frequency in mobile environments. In International Conference on Usability and Internationalization (pp. 646-652). Springer Berlin Heidelberg.

[8] Tongue, Benson, (2001) Principles of Vibration, Oxford Press.

[9] Giuseppe et al (2012) "Small fibre neuropathy." Current opinion in neurology $25.5,542-549$ 